\title{
FCGR3A gene polymorphisms may correlate with response to frontline R-CHOP therapy for diffuse large B-cell lymphoma
}

Dong Hwan Kim, Hee Du Jung, Jong Gwang Kim, Je-Jung Lee, Deok-Hwan Yang, Yeon Hee Park, Young Rok Do, Ho Jin Shin, Min Kyoung Kim, Myung Soo Hyun, and Sang Kyun Sohn

The precise mechanism of rituximab plus cyclophosphamide/doxorubicin/vincristine/prednisone (R-CHOP) therapy in diffuse large $B$-cell lymphoma (DLBCL) is not fully elucidated. Besides overcoming bcl-2-mediated chemoresistance, antibody-dependent cellular cytotoxicity (ADCC), which is activated by effector cells via immunoglobulin G (IgG) fragment $\mathrm{C}$ receptors (FcRs), was also proposed as a mechanism of rituximab. The current study evaluated the impact of FcR polymorphism on the response to $R$ CHOP therapy for DLBCL with the basis that FcR polymorphism can affect rituximab's affinity for ADCC effector cells. The FCGR3A and FCGR2A gene polymorphisms were determined in DLBCL patients receiving $\mathrm{R}-\mathrm{CHOP}(\mathrm{n}=113) \mathrm{com}$ pared with CHOP therapy $(n=85)$. The FCGR3A valine (V) allele was significantly correlated with a higher complete response rate to R-CHOP compared with the phenylalanine $(\mathrm{F})$ allele $(88 \%$ in $\mathrm{V} / \mathrm{V}$ vs $79 \%$ in V/F vs $50 \%$ in $\mathrm{F} / \mathrm{F} ; \boldsymbol{P}=.002$ ), while no difference was found between FCGR2A polymorphisms. In addition, V/V allele was associated with faster achievement of response than other alleles. The impact of the FCGR3A gene polymorphism on response rate was not noted in the CHOP group. In terms of overall or eventfree survival, no difference was found according to FCGR3A or FCGR2A alleles. The FCGR3A single nucleotide polymorphism (SNP) is predictive of response to R-CHOP, but does not correlate with survival in patients with DLBCL. (Blood. 2006; 108:2720-2725)

(C) 2006 by The American Society of Hematology

\section{Introduction}

The introduction of rituximab plus cyclophosphamide/doxorubicin/ vincristine/prednisone (R-CHOP) chemotherapy has significantly improved the treatment outcome of diffuse large B-cell lymphoma (DLBCL). ${ }^{1}$ However, not all patients with DLBCL respond to R-CHOP chemotherapy. Although rituximab has been shown to enhance antibodydependent cellular cytotoxicity (ADCC) in follicular lymphoma (FL), ${ }^{2}$ the mechanism of action of R-CHOP in DLBCL has not been clearly defined. Mounier et $\mathrm{al}^{3}$ have suggested that rituximab possesses synergistic activity with $\mathrm{CHOP}$ that can overcome bcl-2-mediated chemoresistance. However, rituximab also appears to have an antiDLBCL effect by itself. A phase 1 study of rituximab therapy in combination with interleukin-12 (IL-12) in B-cell non-Hodgkin lymphoma (NHL) revealed that the overall response rate was 64\% (7 of 11 patients) with 5 complete remissions (CRs) in CD20-positive DLBCL patients who were previously treated with anthracycline-containing chemotherapy. ${ }^{4}$ These observations would suggest that a different mechanism of action rather than overcoming bcl-2-mediated chemoresistance may be involved in the effect of single-agent rituximab, and that it may be independent of CHOP.

While the precise mechanism of action of rituximab has not been fully elucidated, several theories have been proposed, including ADCC, complement-dependent cytotoxicity (CDC), and induc- tion of apoptosis. ${ }^{5}$ Rituximab is a chimeric monoclonal antibody $(\mathrm{mAb})$ against the CD20 antigen. It has a fragment ab (Fab) domain that binds to the CD20 antigen on B lymphocytes and a fragment $\mathrm{c}$ $(\mathrm{Fc})$ domain that recruits immune effector cells to mediate B-cell lysis through ADCC. ${ }^{6}$

Several types of FcR have been discovered, including Fc $\gamma$ RI,

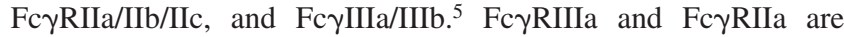
activating receptors due to the presence of an immunoreceptor tyrosine-based activation motif either in the accessory signaling $\gamma$ chain (FcyRIIIa) or cytoplasmic domain (FcyRIIa). Fc $\gamma$ RIIIa is expressed on macrophages, natural killer (NK) cells, and some dendritic cells, while FcyRIIa is expressed on macrophages, neutrophils, dendritic cells, and some mast cells. Previous studies that have examined single nucleotide polymorphisms (SNPs) of Fc receptor $(F c R)$ genes demonstrated that $F C G R 3 A$ gene SNPs are associated with response to rituximab in patients with FL. ${ }^{7}$ This is in contrast to patients with chronic lymphocytic leukemia, ${ }^{8}$ and these differences in response to rituximab are correlated with binding affinity of FcR with ADCC effector cells, suggesting a more important role of ADCC in lymphoma. ${ }^{9-11}$ It is known that the FCGR3A of valine (V) allele has a higher affinity to human immunoglobulin $\mathrm{G}(\mathrm{IgG})$ than the phenylalanine (F) allele, and that
From the Departments of Hematology/Oncology and Laboratory Medicine, Kyungpook National University Hospital, Daegu, Korea; Department of Hematology/Oncology, Samsung Medical Center, Sungkyunkwan University School of Medicine, Seoul, Korea; Department of Hematology/Oncology, Chonnam National University Hwasun Hospital, Jeollanamdo, Korea; Department of Hematology/Oncology, Korean Cancer Center Hospital, Seoul, Korea; Department of Hematology/Oncology, Keimyung University, Dongsan Medical Center, Daegu, Korea; Department of Hematology/Oncology, Pusan National University Hospital, Busan, Korea; and Department of Hematology/Oncology, Yeungnam University, Yeungnam Medical Center, Daegu, Korea.

Submitted January 26, 2006; accepted March 28, 2006. Prepublished online as Blood First Edition Paper, April 11, 2006; DOI 10.1182/blood-2006-01-009480.
Supported by BioMedical Research Institute grant, Kyungpook National University Hospital (2005).

The online version of this article contains a data supplement.

Reprints: Dong Hwan Kim, Department of Hematology/Oncology, Samsung Medical Center, Sungkyunkwan University School of Medicine, Ilwon-dong 50, Kangnam-gu, Seoul, Korea 135-710; e-mail: drkiim@medimail.co.kr.

The publication costs of this article were defrayed in part by page charge payment. Therefore, and solely to indicate this fact, this article is hereby marked "advertisement" in accordance with 18 U.S.C. section 1734.

C 2006 by The American Society of Hematology 
cells bearing the FCGR3A V allele mediate ADCC more effectively. ${ }^{12,13}$

In this study, we evaluated the impact of FCGR3A or FCGR2A gene SNPs on the outcomes of primary R-CHOP therapy in 113 patients with DLBCL, including treatment response and overall and event-free survivals, compared with its contribution on clinical outcomes of CHOP therapy in 85 patients with DLBCL.

\section{Patients, materials, and methods}

\section{Patient characteristics and treatment protocol}

A total of 113 patients who received R-CHOP chemotherapy between April 2002 and March 2005 as a frontline regimen for CD20-positive DLBCL were included in this retrospective study from 6 hospitals in Republic of Korea (Kyungpook National University Hospital, Chonnam National University Hwasun Hospital, Korean Cancer Center Hospital, Dongsan Medical Center, Pusan National University Hospital, and Yeungnam Medical Center). A control group was created from 85 patients who received CHOP therapy as primary chemotherapy for CD20-positive DLBCL between October 1991 and September 2003 who had available paraffin-embedded tissue samples and who had provided informed consent for future research. This study was approved by the institutional research board at the Kyungpook National University Hospital (Daegu, Korea).

The baseline characteristics of the patients are summarized in Table 1. Overall, among 113 patients (median age, 60 years; 64 men, 49 women), 45 (40\%) patients had stages 3 and 4 disease and 37 (33\%) patients had intermediate-to-high or high International Prognostic Index (IPI) scores. R-CHOP followed by involved-field radiation was given to $37(33 \%)$ patients, while R-CHOP alone was given to 76 (67\%) patients. A median of 6 cycles of R-CHOP therapy was given (range, 1-8 cycles). The comparative information between the R-CHOP group and the CHOP group is shown in Table S1 (available on the Blood website; see the Supplemental Materials link at the top of the online article).
R-CHOP chemotherapy was administered as follows: one course of chemotherapy consisted of an intravenous infusion of cyclophosphamide $750 \mathrm{mg} / \mathrm{m}^{2}$, adriamycin $50 \mathrm{mg} / \mathrm{m}^{2}$, vincristine $2 \mathrm{mg}$, and oral administration of $100 \mathrm{mg}$ prednisone on days 1 to 5 (CHOP), which was repeated every 3 weeks. Rituximab $375 \mathrm{mg} / \mathrm{m}^{2}$ was infused over 4 to 6 hours at day 1 before CHOP chemotherapy began. Patients with stage 1 or 2 disease typically received 4 courses of chemotherapy followed by involved-field radiotherapy (30-40 Gy), while patients with advanced stage disease received 6 to 8 courses of chemotherapy followed by radiotherapy to bulky sites. Maintenance treatment with rituximab after completion of R-CHOP therapy was not administered. Of the 113 patients, 5 received high-dose chemotherapy with autologous stem cell rescue due to relapse after response $(\mathrm{n}=3)$ or initially high IPI score $(n=2)$. The response to R-CHOP therapy was evaluated after completion of 2 to 3 courses of R-CHOP chemotherapy and 1 month after completion of all planned cycles of R-CHOP, then every 4 months.

\section{FCGR3A/FCGR2A gene polymorphism study}

Two single-nucleotide polymorphisms of $F C R$ genes were evaluated in the current study involving the FCGR3A and FCGR2A genes as previously described $^{7,14}$ : peripheral blood or bone marrow samples derived from patients were taken before treatment with informed consent. Genomic DNA was extracted from the blood samples using the Wizard genomic DNA purification kit (Promega, Madison, WI). For the control group, paraffinembedded tissue samples were used for DNA purification and only FCGR3A genotyping had been performed.

Two $F c R$ gene polymorphisms were detected based on polymerase chain reactions (PCRs) using primers amplifying a short fragment of DNA containing the polymorphic sites. The first PCR primers for the FCGR3A gene SNP at locus 158 were $5^{\prime}$-ATA TTT ACA GAA TGG CAC AGG -3' and 5'-GAC TTG GTA CCC AGG TTG AA-3', while the second PCR primers for the FCGR3A gene at locus 158 were 5'-ATC AGA TTC GAT CCT ACT TCT GCA GGG GGC AT-3' and 5'-ACG TGC TGA GCT TGA GTG ATG GTG ATG TTC AC-3', generating a 94-bp fragment. The PCR primers for the FCGR2A gene at locus 131 were 5'-GGA AAA TCC CAG

Table 1. Patient characteristics and treatment outcomes according to FCGR3A and FCGR2A alleles

\begin{tabular}{|c|c|c|c|c|c|c|c|c|}
\hline & \multicolumn{3}{|c|}{ FCGR3A } & \multirow[b]{2}{*}{$\boldsymbol{P}$} & \multicolumn{3}{|c|}{ FCGR2A } & \multirow[b]{2}{*}{$P$} \\
\hline & FF & FV & vV & & $\mathrm{HH}$ & HR & RR & \\
\hline No. patients (\%) & $6(5)$ & $54(48)$ & $53(47)$ & & $60(56)$ & $40(37)$ & $8(7)$ & \\
\hline Sex, no. women/men & $3 / 3$ & $27 / 27$ & $19 / 34$ & NS & $25 / 35$ & $16 / 24$ & $5 / 3$ & NS \\
\hline Age, median y (range) & $55(45-76)$ & $60(25-83)$ & $61(18-84)$ & NS & $61(18-82)$ & $60.5(25-84)$ & $60(24-83)$ & NS \\
\hline \multicolumn{9}{|l|}{ Disease, no (\%) } \\
\hline Stages 3,4 & $2(33)$ & $21(39)$ & $22(41)$ & NS & $23(38)$ & $17(43)$ & $3(38)$ & NS \\
\hline Elevated LDH & $2(33)$ & $29(54)$ & $27(51)$ & NS & $33(55)$ & $19(48)$ & $3(38)$ & NS \\
\hline Extranodal & $2(33)$ & $22(41)$ & $25(47)$ & NS & $32(68)$ & $13(28)$ & $2(25)$ & .066 \\
\hline Age, at least $60 \mathrm{y}$ & $2(33)$ & $29(54)$ & $28(53)$ & NS & $32(53)$ & $21(53)$ & $4(50)$ & NS \\
\hline ECOG of at least 2 & $2(33)$ & $10(19)$ & $8(15)$ & NS & $10(17)$ & $9(23)$ & $1(13)$ & NS \\
\hline \multicolumn{9}{|l|}{ IPI score, no. (\%) } \\
\hline $0-2$ & $5(83)$ & $36(67)$ & $34(65)$ & NS & $39(66)$ & $27(67)$ & $6(62)$ & NS \\
\hline $3-5$ & $1(17)$ & $18(33)$ & $18(35)$ & & $20(34)$ & $13(33)$ & $3(38)$ & \\
\hline \multicolumn{9}{|l|}{ Regimen } \\
\hline R-CHOP, no. (\%) & $4(67)$ & $40(74)$ & $32(60)$ & NS & $41(68)$ & $26(65)$ & $5(63)$ & NS \\
\hline R-CHOP + IFRT, no. (\%) & $2(33)$ & $14(26)$ & $21(40)$ & & $19(32)$ & $14(35)$ & $3(37)$ & \\
\hline Cycles, R-CHOP, no. (range) & $5(2-8)$ & $6(2-8)$ & $6(1-8)$ & NS & $6(1-8)$ & $6(1-8)$ & $5(2-6)$ & NS \\
\hline \multicolumn{9}{|l|}{ Response, no. (\%) } \\
\hline Evaluable & $6(100)$ & $53(98)$ & $51(96)$ & & $59(98)$ & $38(95)$ & $8(100)$ & \\
\hline $\mathrm{CR}$ & $3(50)$ & $42(79)$ & $45(88)$ & $.002^{*}$ & $52(88)$ & $29(76)$ & $5(63)$ & .178† \\
\hline PR & $0(0)$ & $6(11)$ & $5(10)$ & & $4(7)$ & $6(16)$ & $1(13)$ & \\
\hline SD / PD & $3(50)$ & $5(9)$ & $1(2)$ & & $3(5)$ & $3(8)$ & $2(25)$ & \\
\hline ORR (CR + PR) & $3(50)$ & $48(90)$ & $50(98)$ & $<.001$ & $56(95)$ & $35(92)$ & $6(75)$ & .137 \\
\hline \multicolumn{9}{|l|}{ Survival, no. (\%) } \\
\hline Relapse & $3(50)$ & $7(13)$ & $12(22)$ & NS & $12(20)$ & $6(15)$ & $2(25)$ & NS \\
\hline Death & $1(17)$ & $5(9)$ & $7(13)$ & NS & $6(10)$ & $4(10)$ & $1(13)$ & NS \\
\hline
\end{tabular}

NS indicates not significant; LDH, lactate dehydrogenase; IFRT, involved-field radiation therapy; SD, stable disease; PD, progressive disease. ${ }^{*} P=.057$ when comparing $\mathrm{CR}$ with non-CR. $\dagger P=.112$ when comparing $\mathrm{CR}$ with non-CR. 
AAA TTC TCG C- $3^{\prime}$ and $5^{\prime}$-CAA CAG CCT GAC TAC CTA TTA CGC GGG-3'. The amplifications were performed on a GeneAmp PCR System 9600 (Applied Biosystems, Foster City, CA) in a $20 \mu \mathrm{L}$ reaction volume containing $100 \mathrm{ng}$ genomic DNA, $10 \mathrm{pmol}$ of each primer, and $\mathrm{Q}$ water (Merck, Darmstadt, Germany) treated with 0.1\% DEPC (Amresco, Solon, $\mathrm{OH})$ using AccuPower PCR Premix (Bioneer, Daejeon, Korea). The PCR program for first-step amplification for the FCGR3A gene at locus 158 consisted of 35 cycles at $95^{\circ} \mathrm{C}$ for 60 seconds, $57^{\circ} \mathrm{C}$ for 90 seconds, $72^{\circ} \mathrm{C}$ for 90 seconds, and a final elongation step at $72^{\circ} \mathrm{C}$ for 8 minutes; second-step amplification for the FCGR3A gene at locus 158 consisted of 35 cycles at $95^{\circ} \mathrm{C}$ for 60 seconds, $64^{\circ} \mathrm{C}$ for 60 seconds, $72^{\circ} \mathrm{C}$ for 60 seconds, and a final elongation step at $72^{\circ} \mathrm{C}$ for 9.5 minutes. The PCR program for the FCGR2A gene at locus 131 consisted of 35 cycles at $94^{\circ} \mathrm{C}$ for 15 seconds, $55^{\circ} \mathrm{C}$ for 30 seconds, $72^{\circ} \mathrm{C}$ for 40 seconds, and a final elongation step at $72^{\circ} \mathrm{C}$ for 7 minutes. The PCR products were checked on a $2 \%$ agarose gel and then subjected to a restriction fragment-length polymorphism (RFLP) analysis.

To distinguish the SNPs, the restriction enzyme NlaIII (New England BioLabs, Beverly, MA) was used for the FCGR3A gene at locus 158, and $B s t \mathrm{UI}$ (New England BioLabs) for the FCGR2A gene at locus 131. Each $5 \mu \mathrm{L}$ of the PCR products was digested for 2 hours with $5 \mathrm{U}$ NlaIII (FCGR3A gene at locus 158 , at $60^{\circ} \mathrm{C}$ ), and Bst $\mathrm{UI}(F C G R 2 A$ gene at locus 131 , at $37^{\circ} \mathrm{C}$ ). The digested products were separated on a $2 \%$ low-meltingtemperature agarose gel (Cambrex Bio Science Rockland, Rockland, ME) and stained with ethidium bromide. For the FCGR3A gene at locus 158, the $\mathrm{V}$ allele was identified as 2 digested bands at $61 \mathrm{bp}$ and $33 \mathrm{bp}$, while the $\mathrm{F}$ allele was identified as one undigested band at $94 \mathrm{bp}$. For the FCGR2A gene at locus 131, the histidine $(\mathrm{H})$ allele was identified as a 337-bp DNA band, while the arginine $(\mathrm{R})$ allele was identified as a 316-bp and 21-bp band. Then, selected PCR-amplified DNA samples were examined by DNA sequencing to confirm the genotyping results.

\section{Definitions}

Clinical responses to R-CHOP chemotherapy were determined by physical examination and computed tomography before starting subsequent radiation therapy once patients were planned to receive involved-field radiation. The responses were scored according to International Working Group criteria. ${ }^{15}$ Overall survival (OS) was measured from day 1 of the first cycle of R-CHOP until the date of death or last follow-up. Event-free survival (EFS) was calculated from day 1 of the first cycle of R-CHOP until treatment failure (disease progression, recurrence, or death of any cause). To exclude potential effects of high-dose chemotherapy with autologous stem cell rescue on survival, patients were considered as censoring cases at the time of transplantation. The time to response to R-CHOP therapy was calculated as an interval between day 1 of the first cycle of R-CHOP and the day response was confirmed, including complete and partial responses.

\section{Statistical analysis}

Clinical data were analyzed according to information available as of November 2005. The primary objective of the current study was to correlate FCGR3A/FCGR2A SNPs with the response to R-CHOP therapy. The secondary objectives were to correlate FCGR3A/FCGR2A SNPs with survival outcomes including OS and EFS, and with the time to response to R-CHOP therapy. In addition, the correlation between FCGR3A SNP and FCGR2A SNP was also analyzed in the current study.

The clinical characteristics and treatment outcomes of the patients were compared using Chi-square, Fisher exact, or Mann-Whitney $U$ tests according to the FCGR3A/FCGR2A SNPs. The genetic association between FCGR3A and FCGR2A gene SNPs was determined using the Chi-square test. The clinical characteristics and the response to primary chemotherapy were compared according to the FCGR3A/FCGR2A SNPs in the R-CHOP group and $\mathrm{CHOP}$ group, respectively. Logistic regression analysis was conducted to determine the predictive value of the SNPs on response to R-CHOP for 105 patients who had both FCGR3A and FCGR2A SNP data available. Variables included for the logistic regression were stage (stages 1 and 2 vs 3 and 4), IPI score ( $0-2$ vs $3-5)$, age ( $<60$ years vs $\geq 60$ years), performance status (Eastern Cooperative Oncology Group [ECOG] 0,1 vs $\geq 2$ ), lactate dehydrogenase level (normal vs beyond elevated), extranodal involvement ( $\leq 1$ site vs $\geq 2$ sites), and $F C G R 3 A$ (V/V allele vs non-V/V allele) and FCGR2A (H/H allele vs non-H/H allele) alleles. Odds ratio (OR) and $95 \%$ confidence intervals (CIs) for relative risks were also calculated by logistic regression analysis.

Survival estimates were calculated using the Kaplan-Meier method. Differences in OS, EFS, and the time to response to R-CHOP therapy according to the FCGR3A/FCGR2A SNPs were compared using log-rank tests or a Wilcoxon test (for the time to response to R-CHOP therapy). For the multivariate survival analyses using the Cox proportional hazard model to define the prognostic factors for OS, EFS, and the time to response to R-CHOP therapy, a backward conditional procedure was conducted until the $P$ value for the likelihood ratio test was above .05 with the following variables: stage (stages 1,2 vs 3,4$)$, IPI score ( $0-2$ vs $3-5)$, age $(<60$ years vs $\geq 60$ years), performance status (ECOG 0 or 1 vs $\geq 2$ ), lactate dehydrogenase level (normal vs beyond normal range), extranodal involvement ( $\leq 1$ site vs $\geq 2$ sites), and $F C G R 3 A$ (V/V allele vs non-V/V allele) and $F C G R 2 A(\mathrm{H} / \mathrm{H}$ allele vs non-H/H allele) allele. The hazard ratio (HR) and $95 \%$ CI were also estimated. A cut-off $P$ value of .05 was adopted for all the statistical analyses. The statistical data were obtained using an SPSS software package, version 11.5 (SPSS, Chicago, IL). Multivariate analyses were performed on 105 of 113 patients; Fc $\gamma$ RIIa allele data was unavailable for 8 patients.

\section{Results}

\section{Frequency of FCGR3A and FCGR2A alleles}

In the R-CHOP group, the distribution of the VV, VF, and FF alleles of FCGR3A was $47 \%, 48 \%$, and $5 \%$, respectively, while the distribution of HH, HR, and RR alleles in FCGR3A was $56 \%, 37 \%$, and $7 \%$, respectively. No significant correlation between FCGR3A and FCGR2A alleles was demonstrated $(P=.142$ by $\chi$-square test). The distribution of VV, VF, and FF alleles of FCGR3A in the CHOP group was $57 \%, 31 \%$, and $12 \%$, respectively (Table S1).

\section{Patient characteristics according to FCGR3A and FCGR2A allele status}

The patients' characteristics are summarized in Table 1. In brief, no differences in patient and disease characteristics were observed more frequently according to FCGR3A and FCGR2A alleles, except for extranodal DLBCL involvement, which was more common with the FCGR $2 A \mathrm{H} / \mathrm{H}$ allele. In addition, except for sex discrepancy, no differences in patient and disease characteristics were observed more frequently according to FCGR3A and FCGR2A alleles in the CHOP group (Table S1).

\section{Response to frontline R-CHOP therapy according to FCGR3A and FCGR2A alleles}

Of 110 patients evaluable for response to chemotherapy, the overall response rate (ORR) was $92 \%$ (101 of 110 patients) with a CR of $82 \%$ (90 of 110 patients), and a partial response (PR) rate of $10 \%$ (11 of 110 patients). As shown in Table 1 and Figure 1, a higher CR rate was observed in the $F C G R 3 A \mathrm{~V} / \mathrm{V}$ allele ( $88 \%$ ) compared with the FCGR3A V/F $(79 \%)$ or $\mathrm{F} / \mathrm{F}$ allele $(50 \% ; P=.002)$. The difference in ORR was also significant in favor of the FCGR3A V/V allele (ORR of $98 \%$ vs FCGR3A V/F [90\%] or F/F allele $[50 \%] ; P<.001)$. A statistically significant difference in response rate was not observed according to FCGR $2 A$ allele even though the trend of better response rate was observed in FCGR $2 A \mathrm{H} / \mathrm{H}$ allele (95\% ORR vs FCGR2A H/R [92\%] or R/R allele [75\%]; $P=.137)$. 

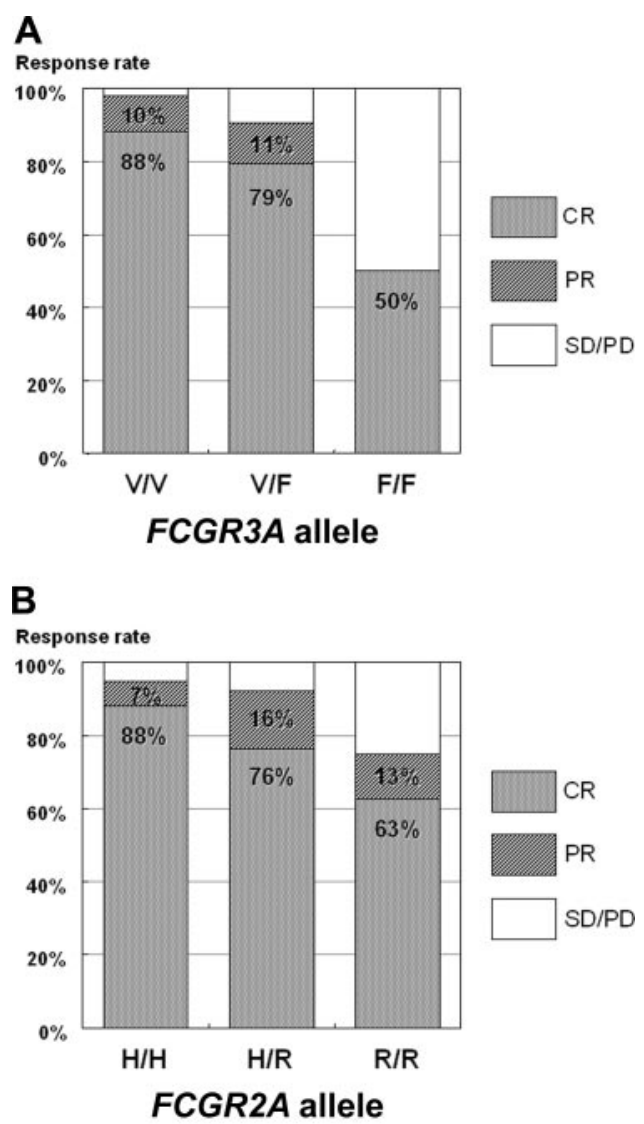

Figure 1. Response to frontline R-CHOP therapy according to FCGR3A and FCGR2A alleles. (A) $P=.002 . P=.001$ when $\mathrm{CP}$ and $\mathrm{PR}$ are compared with DC and PD. (B) $P=.178$.

When comparing the response to frontline chemotherapy between the R-CHOP and CHOP groups, it was significantly different in favor of the R-CHOP group with respect to an achievement of OR $(91 \%$ vs $70 \% ; P<.001)$ or $\mathrm{CR}(81 \%$ vs $53 \%, P<.001$; Figure S1A). Meanwhile, the impact of the FCGR3A V/V allele on OS or $\mathrm{CR}$ rates was not noted in the CHOP group as shown in Table $\mathrm{S} 1$ and Figure S1B.

When analyzing the time to CR with R-CHOP (Figure 2A), we found that the group with the $F C G R 3 A \mathrm{~V} / \mathrm{V}$ allele had a more rapid time to $\mathrm{CR}$ (median, 70 days; $95 \%$ CI, 52-88 days) compared with non-V/V allele (median, 99 days, $95 \%$ CI $60-138$ days; $P=.020$ by Wilcoxon test). A similar trend was also observed in terms of the time to achievement to OR (Figure 2B) with a more rapid response in the group with the FCGR3A V/V allele (median, 64 days; 95\% CI 52-76 days) versus non-V/V allele (median, 77 days, $95 \%$ CI 74-80 days; $P=.013$ by Wilcoxon test). However, a significant influence of the FCGR2A allele was not observed on the time to achievement of $\mathrm{CR}(P=.515)$ or $\mathrm{OR}(P=.789)$.

\section{Survival analysis according to FCGR3A and FCGR2A allele}

With a median follow-up duration of 420 days (range, 12-1297 days), 22 (19\%) patients had relapsed or progressed, with $13(12 \%)$

Table 2. Logistic regression analysis for response to frontline R-CHOP therapy

\begin{tabular}{lccc}
\hline FCGR3A allele & Response rate, $\%$ & OR $(95 \%$ Cl $)$ & $\boldsymbol{P}$ \\
\hline V/V & 98 & $7.686(1.028-61.748)$ & .048 \\
Non-V/V & 86 & 1.000 & \\
\hline
\end{tabular}

Table 3. Multivariate analysis for achievement of complete response after frontline R-CHOP therapy

\begin{tabular}{lccc}
\hline & $\begin{array}{c}\text { Median time to } \\
\text { response, d }\end{array}$ & HR (95\% Cl) & $\boldsymbol{P}$ \\
\hline $\begin{array}{l}\text { FCGR3A allele } \\
\text { V/V }\end{array}$ & 70 & $1.654(1.076-2.542)$ & .022 \\
Non-V/V & 99 & 1.000 & \\
$\begin{array}{l}\text { Stage } \\
\text { I, II }\end{array}$ & 79 & $1.640(1.033-2.603)$ & .036 \\
III, IV & 130 & 1.000 & \\
IPI score & & & \\
0-2 & 82 & $2.764(1.255-6.088)$ & .012 \\
3-5 & 111 & 1.000 & \\
\hline
\end{tabular}

deaths. The 1- and 2-year OS rate was $93 \% \pm 3 \%$ and $82 \% \pm 5 \%$, while $1-$ and 2-year EFS rates were $83 \% \pm 4 \%$ and $70 \% \pm 6 \%$, respectively. When comparing OS and EFS according to FCGR3A and FCGR2A alleles, neither the FCGR3A nor the FCGR2A allele had any impact on OS or EFS (Figure 3).

\section{Multivariate analysis}

The results of the multivariate analysis are summarized in Tables 2-4. When analyzing predictive factors for response to R-CHOP therapy, the FCGR3A V/V allele was found to be an independent factor predicting OR; patients with the FCGR3A V/V allele were found to have a more than 7-fold higher probability of OR $(P=.048$, HR $7.686,95 \%$ CI 1.028-61.748). In addition, the FCGR3A V/V allele was also identified as a predictive factor for rapid achievement of $\mathrm{CR}(P=.022$, HR $1.654,95 \%$ CI $1.076-$ $2.542)$, together with early-stage disease $(P=.036$, HR 1.640 , $95 \%$ CI $1.033-2.603)$ and low IPI score $(P=.012$, HR $2.764,95 \%$ CI 1.255-6.088). A similar outcome was also identified on the analysis for the time to OR: the FCGR $3 A \mathrm{~V} / \mathrm{V}$ allele $(P=.005, \mathrm{HR}$ $1.802,95 \% \mathrm{CI} 1.193-2.723)$ and early-stage disease $(P=.050, \mathrm{HR}$ $1.522,95 \%$ CI $1.004-2.314)$.

However, in a Cox multivariate analysis of survival, neither the FCGR3A nor FCGR2A alleles were independently associated with survival after R-CHOP therapy for DLBCL. On the other hand, low IPI score was found to be a favorable prognostic factor for OS $(P=.007$, HR $0.199,95 \%$ CI $0.061-0.646)$ and EFS $(P<.001$, HR $0.107,95 \%$ CI $0.042-0.271)$ after R-CHOP therapy for patients with DLBCL.

\section{Discussion}

This study demonstrates that the FCGR3A SNP is predictive of response to R-CHOP chemotherapy in patients with DLBCL, but is not predictive of OS or EFS. Based on the current observation, the additive effect of rituximab through ADCC, which is mediated via FcR, may be one of the important action mechanisms of R-CHOP in patients with DLBCL in addition to

Table 4. Multivariate analysis for EFS and OS after frontline R-CHOP therapy

\begin{tabular}{lccc}
\hline Risk factor & 2-y survival rate, $\%$ & HR $(95 \% \mathrm{Cl})$ & $\boldsymbol{P}$ \\
\hline OS & & & \\
IPI score 0-2 & $93.1 \pm 3.4$ & $0.199(0.061-0.646)$ & .007 \\
IPI score 3-5 & $59.7 \pm 11.7$ & 1.000 & \\
EFS & & & \\
IPI score 0-2 & $84.7 \pm 6.8$ & $0.107(0.042-0.271)$ & $<.001$ \\
IPI score 3-5 & $38.8 \pm 10.9$ & 1.000 & \\
\hline
\end{tabular}


A

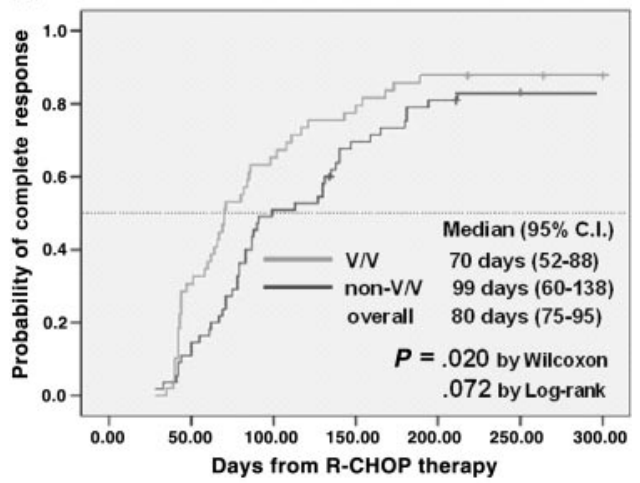

B

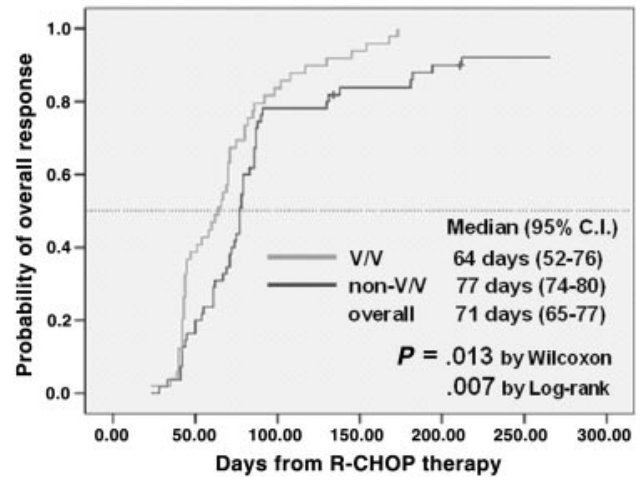

Figure 2. The cumulative incidence of achievement of $C R$ and OR to frontline $\mathrm{R}-\mathrm{CHOP}$ therapy according to FCGR3A allele.

the synergistic activity of rituximab with CHOP by overcoming bcl-2-mediated chemoresistance.

Although the precise mechanism of action of rituximab is not fully elucidated, it likely includes ADCC, complement-dependent cytotoxicity, and induction of apoptosis. ${ }^{5}$ The benefit of R-CHOP therapy has been demonstrated in a randomized trial of R-CHOP versus CHOP in elderly patients with $\mathrm{DLBCL}^{1}$; in that trial, the benefit of rituximab was primarily limited to patients with overexpression of bcl-2, suggesting that rituximab may act by overcoming bcl-2-mediated chemoresistance in part. ${ }^{3}$ This observation was supported by Chow et al, ${ }^{16}$ who demonstrated that combining rituximab with different cytotoxic drugs such as doxorubicin or mitoxantrone significantly decreased the dose of chemotherapeutic drugs necessary for the induction of apoptosis.

However, several investigators have suggested that the mechanism of action of rituximab is not solely confined to overcoming bcl-2-mediated chemoresistance. Ansell et al ${ }^{4}$ reported that a high response rate was observed in lymphoma trials when rituximab was combined with nonchemotherapeutic agents such as IL-12, which can enhance cellular cytotoxicity, and that the ORR was $64 \%$ (7 of 11 patients), with 5 CRs in CD20-positive DLBCL patients who were previously treated with anthracycline-containing chemotherapy. This study suggested that tissue macrophages may be critical for the treatment of DLBCL with rituximab treatment as well as for FL. ${ }^{4}$ In addition, Manches et $\mathrm{al}^{17}$ reported that rituximab can induce lymphoma cell phagocytosis by tissue-scavenging cells such as macrophages or NK cells, important mediators of ADCC.

The binding of rituximab to CD20-positive DLBCL cells allows the recruitment of effector cells of ADCC such as NK cells and macrophages, which express $\mathrm{Fc}$ receptor. ${ }^{6} \mathrm{Fc} \gamma \mathrm{RIII}$ is predominantly expressed on NK cells and macrophages, whereas Fc $\gamma$ RIIa is expressed on neutrophils and macrophages. Previous investigations suggested that the FCGR3A/FCGR2A gene SNPs can influence the response to rituximab therapy in $\mathrm{FL}^{2,7}$ and in Waldenström macroglobulinemia. ${ }^{8}$ The FCGR3A/FCGR $2 A$ gene SNPs may influence treatment outcome in several ways. First, the FCGR3A genotype may affect rituximab pharmacokinetics, thus influencing the response rate of R-CHOP. ${ }^{11}$ In addition, NK cell-mediated ADCC may contribute to the action of rituximab on DLBCL cells. After binding to NK cells through FcyRIIIa, granzyme release may activate caspase, thus overcoming chemoresistance of lymphoma cells. ${ }^{16}$

The major polymorphism of FCGR3A is a point mutation that results in the expression in the extracellular domain at amino acid position 158 of either V (V158) or F (F158). The FCGR3A-V158 genotype has a higher affinity for IgG than does the F158 genotype ${ }^{18}$ Recent investigations also suggested that the individuals expressing FCGR3A-158 VV and VF showed increased NK cell-surface expression of Fc $\gamma$ RIIIa and binding of rituximab, and demonstrated higher levels of ADCC activity in response to rituximab in healthy individuals. ${ }^{19}$

Our results demonstrate that FCGR3A SNPs correlate with the response to R-CHOP in patients with DLBL (especially the V allele for better and faster response; Figures 1-2). The same phenomenon was not observed in the CHOP group without any correlation of the FCGR3A SNPs to response to CHOP chemotherapy. These observations imply that ADCC may contribute in part to the mechanism of R-CHOP. However, no association of FCGR3A SNPs with survival was noted in the present study, for the following reasons: (1) the relatively small number of patients; (2) the relatively short period of follow-up may not be enough to see significant difference of survival; and (3) ADCC itself may not be a predominant mechanism of R-CHOP in DLBCL patients in terms of survival. Other mechanisms such as bcl-2-mediated chemoresistance are
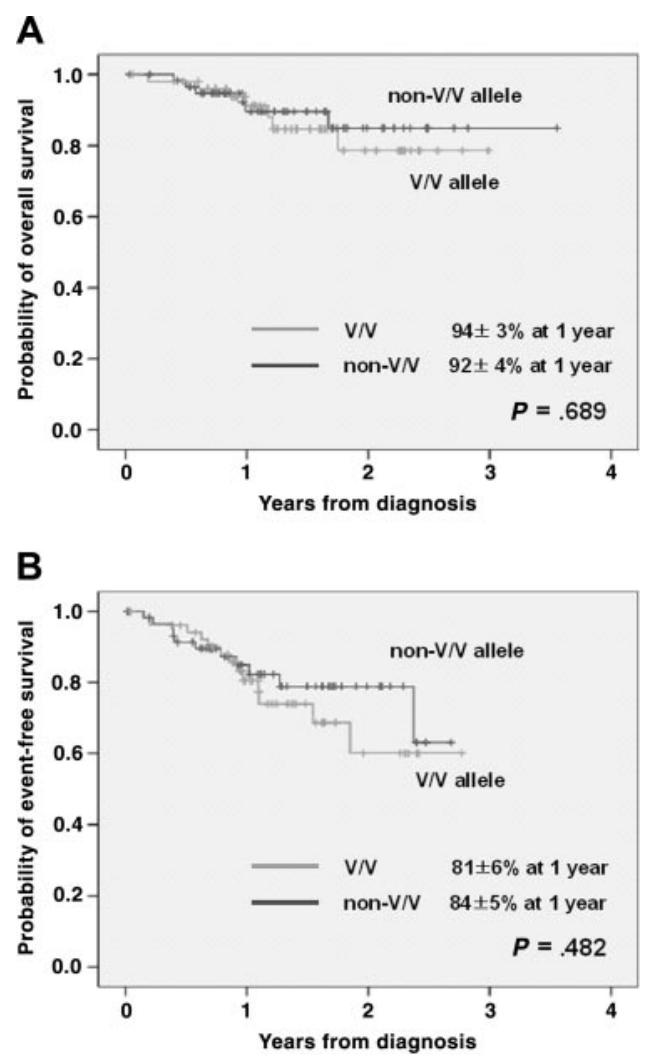

Figure 3. Survival curve after frontline R-CHOP therapy according to FCGR3A allele. 
likely important in the mechanism of action of R-CHOP, and this would be expected to affect survival of patients with DLBCL. Accordingly, further study will be necessary to reach a clear conclusion on this issue, especially on OS or EFS.

The major FCGR2A polymorphism is a point mutation affecting amino acid position 131, coding for either arginine (R131) or histidine (H131). The FCGR2A-H131 genotype has higher affinity for human IgG3. However, we could not determine the role of the $F C G R 2 A$ allele in predicting the response and prognosis in patients with DLBCL who received R-CHOP therapy.

In the current study, the frequency of the $\mathrm{V} / \mathrm{V}$ allele was relatively higher than that of previous reports in the white population. ${ }^{2,7}$ However, the frequency distribution of the Chinese population was very similar to that of our study (48.9\% of patients had the V/V genotype, compared with $47 \%$ in the current study). ${ }^{20}$
The different distribution of allelic frequencies may result from different ethnic backgrounds.

In conclusion, the present study suggests that the FCGR3A SNP is a predictive of response to R-CHOP, but may not be a prognostic factor for patients with DLBCL. Besides the synergistic role of rituximab when combined with $\mathrm{CHOP}$ in overcoming bcl-2mediated chemoresistance, the additive effect of rituximab through ADCC that was mediated by FcR may be one of the important action mechanisms of R-CHOP in DLBCL.

\section{Acknowledgments}

We gratefully give thanks to Dr John Kuruvilla and Dr Grace Wu for their critical review of the manuscript.

\section{References}

1. Feugier $P$, Van Hoof $A$, Sebban $C$, et al. Longterm results of the R-CHOP study in the treatment of elderly patients with diffuse large B-cell lymphoma: a study by the Groupe d'Etude des Lymphomes de l'Adulte. J Clin Oncol. 2005;23: 4117-4126.

2. Cartron G, Dacheux L, Salles G, et al. Therapeutic activity of humanized anti-CD20 monoclonal antibody and polymorphism in IgG Fc receptor FcgammaRIlla gene. Blood. 2002;99:754-758.

3. Mounier N, Briere J, Gisselbrecht C, et al. Rituximab plus $\mathrm{CHOP}$ (R-CHOP) overcomes bcl-2associated resistance to chemotherapy in elderly patients with diffuse large B-cell lymphoma (DLBCL). Blood. 2003;101:4279-4284.

4. Ansell SM, Witzig TE, Kurtin PJ, et al. Phase 1 study of interleukin-12 in combination with rituximab in patients with B-cell non-Hodgkin lymphoma. Blood. 2002;99:67-74

5. Cartron G, Watier H, Golay J, Solal-Celigny P. From the bench to the bedside: ways to improve rituximab efficacy. Blood. 2004;104:2635-2642.

6. Reff ME, Carner K, Chambers KS, et al. Depletion of $B$ cells in vivo by a chimeric mouse human monoclonal antibody to CD20. Blood. 1994;83: 435-445.

7. Weng WK, Levy R. Two immunoglobulin G fragment $C$ receptor polymorphisms independently predict response to rituximab in patients with follicular lymphoma. J Clin Oncol. 2003;21:39403947.
8. Treon SP, Hansen M, Branagan AR, et al. Polymorphisms in FcgammaRIIIA (CD16) receptor expression are associated with clinical response to rituximab in Waldenstrom's macroglobulinemia. J Clin Oncol. 2005;23:474-481.

9. Ernst LK, Metes D, Herberman RB, Morel PA. Allelic polymorphisms in the FcgammaRIIC gene can influence its function on normal human natural killer cells. J Mol Med. 2002;80: 248-257.

10. van Sorge NM, van der Pol WL, van de Winkel JG. FcgammaR polymorphisms: implications for function, disease susceptibility and immunotherapy. Tissue Antigens. 2003;61:189-202.

11. Dall'Ozzo S, Tartas S, Paintaud G, et al. Rituximab-dependent cytotoxicity by natural killer cells: influence of FCGR3A polymorphism on the concentration-effect relationship. Cancer Res. 2004; 64:4664-4669.

12. Wu J, Edberg JC, Redecha PB, et al. A novel polymorphism of FcgammaRIIla (CD16) alters receptor function and predisposes to autoimmune disease. J Clin Invest. 1997;100:1059-1070.

13. Shields RL, Namenuk AK, Hong K, et al. High resolution mapping of the binding site on human IgG1 for Fc gamma RI, Fc gamma RII, Fc gamma RIII, and FcRn and design of IgG1 variants with improved binding to the Fc gamma R. J Biol Chem. 2001;276:6591-6604.

14. Lin TS, Flinn IW, Modali R, et al. FCGR3A and FCGR2A polymorphisms may not correlate with response to alemtuzumab in chronic lymphocytic leukemia. Blood. 2005;105:289-291.

15. Cheson BD, Horning SJ, Coiffier B, et al. Report of an international workshop to standardize response criteria for non-Hodgkin's lymphomas: $\mathrm{NCI}$ Sponsored International Working Group. J Clin Oncol. 1999;17:1244.

16. Chow KU, Sommerlad WD, Boehrer S, et al. AntiCD20 antibody (IDEC-C2B8, rituximab) enhances efficacy of cytotoxic drugs on neoplastic lymphocytes in vitro: role of cytokines, complement, and caspases. Haematologica. 2002;87:33-43.

17. Manches O, Lui G, Chaperot L, et al. In vitro mechanisms of action of rituximab on primary non-Hodgkin lymphomas. Blood. 2003;101:949-954.

18. van der Pol W, van de Winkel JG. IgG receptor polymorphisms: risk factors for disease. Immunogenetics. 1998;48:222-232.

19. Hatjiharissi E, Santo DD, Xu L, et al. Individuals Expressing Fc $\gamma$ IIIA-158 V/V and V/F show increased NK cell surface expression of Fc $\gamma$ RIIIA (CD16), rituximab binding, and demonstrate higher levels of ADCC activity in response to rituximab [abstract]. Blood. 2005;106:776a.

20. Wang J, Feng J, Zhang L, et al. Distribution of variant genotypes of Fc gamma receptor IIla in healthy Chinese population of Zhengzhou city. J Huazhong Univ Sci Technolog Med Sci. 2004;23: 239-241. 


\section{FCGR3A gene polymorphisms may correlate with response to frontline R-CHOP therapy for diffuse large B-cell lymphoma}

Dong Hwan Kim, Hee Du Jung, Jong Gwang Kim, Je-Jung Lee, Deok-Hwan Yang, Yeon Hee Park, Young Rok Do, Ho Jin Shin, Min Kyoung Kim, Myung Soo Hyun and Sang Kyun Sohn

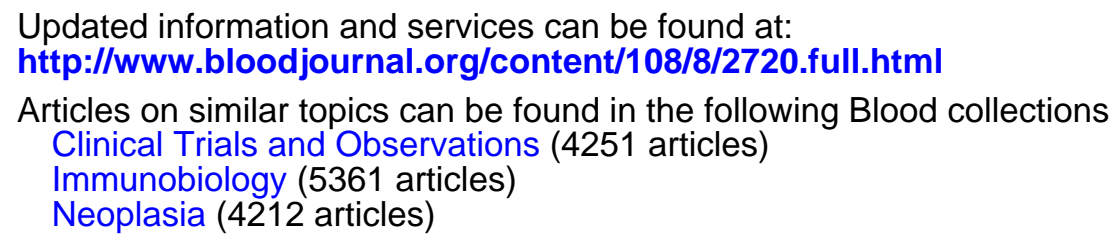

Information about reproducing this article in parts or in its entirety may be found online at: http://www.bloodjournal.org/site/misc/rights.xhtml\#repub_requests

Information about ordering reprints may be found online at:

http://www.bloodjournal.org/site/misc/rights.xhtml\#reprints

Information about subscriptions and ASH membership may be found online at:

http://www.bloodjournal.org/site/subscriptions/index.xhtml 